\title{
Acoustic Radiation Force Impulse (ARFI) Elastography for non-invasive evaluation of hepatic fibrosis in chronic hepatitis $B$ and $C$ patients: a systematic review and meta-analysis
}

\author{
Xiangdong Hu, Lanyan Qiu, Dong Liu, Linxue Qian
}

Department of Ultrasound, Beijing Friendship Hospital, Capital Medical University, Beijing, China

\begin{abstract}
Aim: The purpose of the study was to assess the effect of Acoustic Radiation Force Impulse (ARFI) elastography in the diagnosis of liver fibrosis in chronic hepatitis B and C patients through Meta-analysis. Material and methods: Four databases (PubMed, the Cochrane Library, WanFang data, and CNKI) were searched. The key words were: ("ARFI" or "acoustic radiation force impulse") combined with "liver fibrosis" and ("chronic hepatitis" or "HBV HCV"). Heterogeneity $\left(I^{2}\right)$ was assessed, and its source was analyzed through meta-regression. Results: 21 articles with 2,691 patients were included. The composite $\mathrm{Se}=0.79$ (95\% CI: 0.76-0.83) and $\mathrm{Sp}=0.86$ (95\% CI: 0.85-0.88). ARFI elastography showed a better ability to evaluate higherstage liver fibrosis and liver cirrhosis $\left(\mathrm{F}=3\right.$ and $\mathrm{F}=4$, respectively). For $\mathrm{F} \geq 3, \mathrm{Se}=0.84$ (95\% CI: $\left.0.80-0.88, I^{2}=61.37\right), \mathrm{Sp}=0.90$ (95\% CI: $\left.0.86-0.92, I^{2}=65.10\right)$, and AUROC $=0.94$ (95\% CI: $\left.0.91-0.95\right)$. Se and Sp and AUROC of F=4 were $0.86(95 \% \mathrm{CI}$ : 0.80-0.91, $\left.I^{2}=70.67\right), 0.84$ (95\% CI: $\left.0.80-0.88, I^{2}=78.94\right)$ and 0.91 (95\% CI: 0.89-0.94), respectively. Besides, the combined ARFI values indicate that $\mathrm{CHC}$ patients had higher ARFI values especially in the F3 stage (1.87 [95\% CI: 1.67-2.06] and 2.31 [95\% CI: 2.09-2.52] for CHB and CHC, respectively). Conclusion: ARFI elastography is accurate and reliable in the diagnosis of CHB- and $\mathrm{CHC}$-induced liver fibrosis and is especially suitable for the evaluation of stages $\mathrm{F} \geq 3$ and $\mathrm{F}=4$. $\mathrm{CHC}$ patients manifest higher ARFI values than CHB patients especially in the F3 stage.

Keywords: Acoustic Radiation Force Impulse (ARFI); hepatic fibrosis; chronic hepatitis C; chronic hepatitis B; metaanalysis.
\end{abstract}

\section{Introduction}

Chronic hepatitis B (CHB), caused by the hepatitis B virus (HBV), is one of the most serious and prevalent liver conditions. Globally, more than 350 million chronic hepatitis patients are HBV carriers [1-3]. Similarly to CHB, chronic hepatitis $\mathrm{C}(\mathrm{CHC})$ results from an infection with the hepatitis $\mathrm{C}$ virus (HCV). Worldwide, approximately 150 million people are infected with $\mathrm{HCV}$, and each year

Received 18.05.2016 Accepted 27.08.2016

Med Ultrason

2017, Vol. 19, No 1, 23-31

Corresponding author: Linxue Qian

Department of Ultrasound, Beijing Friendship

Hospital, Capital Medical University, 95

Yong'an Road, Xicheng District, Beijing, 100050, China

Phone: +8613520983647

E-mail: qianlinxue_2016@163.com
3 to 4 million new cases of infection have been reported $[4,5]$. People with $\mathrm{CHB}$ or $\mathrm{CHC}$ have a risk to develop hepatic fibrosis, cirrhosis, and liver failure, or even hepatocellular carcinoma [6-8]. Patients with absent or mild liver fibrosis at diagnosis are considered to have a relatively lower risk $(25 \%-30 \%)$ of progression to cirrhosis over the next 20 years [9]. Thus, the prognosis and treatment decisions often depend on the fibrosis severity, and therefore, the evaluation of liver fibrosis is crucial to the successful therapeutic outcomes [10]. Liver biopsy (LB) is considered the golden standard for liver fibrosis assessment. However, it has some limitations. The primary limitation is the invasiveness of the procedure. Although the damage is "minimal", LB procedure still causes pain and minor or major bleeding $(0.3 \%)$ and can lead to complications even under ideal clinical conditions [11]. In the second place, the accuracy of liver biopsy is limited by intra- and interobserver variability and sampling error [12]. 
In the recent years, some alternative methods, including acoustic radiation force impulse (ARFI) elastography, have received widespread attention. ARFI is a new and promising ultrasound-based diagnostic technique. By short-duration acoustic radiation forces (less than $1 \mathrm{~ms}$ ), the selected region of interest localizes the displacements without any external compression, and thus the operator dependency is reduced. The generated wave scan provides qualitative or quantitative responses (wave velocity and ARFI values) [13].

The effect of ARFI elastography on liver fibrosis has been extensively evaluated, and several meta-analyses have been published, but the debates over the effect of ARFI elastography on liver fibrosis have never stopped. However, some new studies published over the recent years have not been included in the previous meta-analyses. Thus, in this meta-analysis, we have exerted the maximal effort to find and combine every single analysis to evaluate the efficacy of ARFI elastography on liver fibrosis caused by $\mathrm{CHB}$ and $\mathrm{CHC}$.

The purpose of the present review was to broaden the sample size and narrow the error through meta-analysis to assess the overall effect of ARFI in the diagnosis of liver fibrosis in $\mathrm{CHB}$ and $\mathrm{CHC}$ patients.

\section{Material and methods}

\section{Article Search Strategy}

To obtain the maximum possible number of publications, we searched not only PubMed and the Cochrane Library but also the Chinese databases WanFang data and China National Knowledge Infrastructure (CNKI). The key words were: ("ARFI" or "acoustic radiation force impulse") combined with "liver fibrosis" and ("chronic hepatitis" or "HBV HCV"). Only human studies were included, with no time lower limit (up to April 25, 2016).

\section{Inclusion criteria}

The following inclusion criteria were applied: 1) The studies were diagnostic tests and evaluated the effect of ARFI elastography in disease diagnosis in different stages of hepatic fibrosis; 2) The studies focused on CHB and $\mathrm{CHC}$ or on other diseases but contained solo data of CHB or CHC; 3) The patients were adults; 4) The studies provided data of comparisons between ARFI and LB (the golden standard).

\section{Exclusion criteria}

Articles were excluded if they had the following characteristics: 1) No solo data for CHB or CHC; 2) The studies focused on the probe type of ARFI or used spleen ARFI to examine hepatic fibrosis; 3) Animal research was conducted; 4) The publications were case reports, supplements, abstracts, reviews, systematic reviews, and meta-analyses; 5) Data deficiency existed; 6) HIV coinfection was present.

\section{Quality assessment}

The Quality Assessment of Studies of Diagnostic Accuracy (QUADAS-2 tool) was used to assess the Quality of each study [14].

\section{Data extraction}

We extracted the following data from original articles: the author's name, year of publication, patient's country of origin, age, gender ratio, ARFI values and standard error (SE), fibrosis stage (diagnosed by LB), patients' number in each of the different fibrosis stages, as well as the values of the true positive (tp), false positive (fp), false negative (fn), and true negative (tn) detection rates, sensitivity (Se), specificity (Sp), and area under ROC curve (AUROC), and $95 \%$ confidence interval $(95 \% \mathrm{CI})$. The extraction of all data was performed by Microsoft Excel 2007.

\section{Statistical analysis}

The values of tp, fp, fn, and tn of different fibrosis stages had to be calculated if the original data did not provide them. Then, we used the Midas command in Stata 12.1 Meta-analysis model to combine tp, fp, fn, and tn to estimate the combined sensitivities, specificities, and AUROC of the different fibrosis stages. ROC curves were also obtained. Meanwhile, we combined the ARFI values that had been divided by $\mathrm{CHC}$ and $\mathrm{CHB}$ by different fibrosis stages.

$I^{2}$ was used to assess heterogeneity. If $I^{2}>50 \%$, the presence of heterogeneity was indicated. When $I^{2}<50 \%$, we combined the data by the fixed-effect model; otherwise, we used the random-effect model. If heterogeneity existed, we analyzed its source through meta-regression. Report bias was determined by funnel plot. All statistical analyses were performed by Stata 12.1.

\section{Results}

\section{Article selection}

We collected a total of 445 articles: 369 articles of them were obtained after searching PubMed and the Cochrane Library, and 76 Chinese articles were found through a search on Wanfang data and CNKI s. Articles that were repeated or met the exclusion criteria, were excluded. To combine sensitivity, specificity, and AUROC, 21 [15-35] articles with 22 studies and 2,691 patients were included in the diagnosis analysis. Of the 21 articles, $11[15,18,20,22,26-30,33,35]$ articles provided ARFI values that were combined in the analysis. Details are present in figure 1.

\section{Quality assessment}

Articles which were intended to be combined into meta-analysis needed to receive quality assessment by 


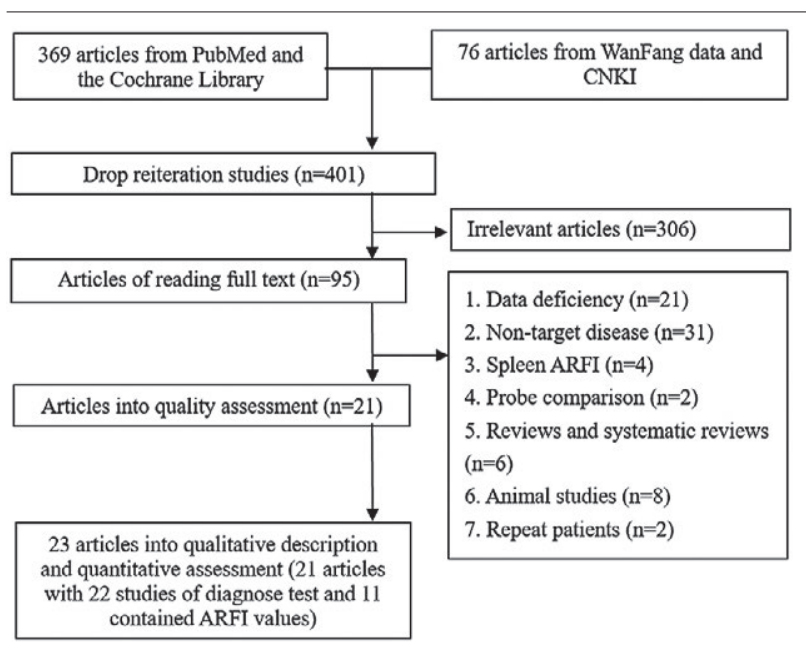

Fig 1. Details of article research.

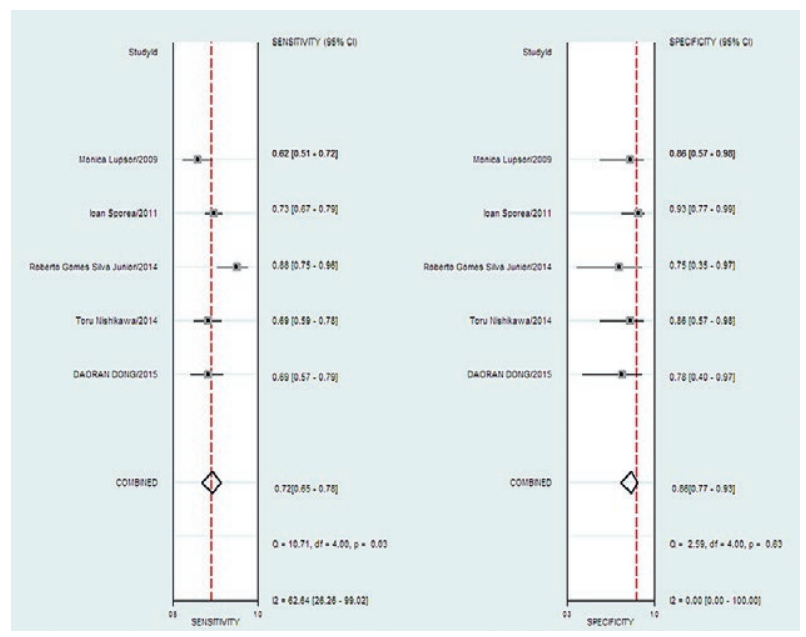

Fig 2. Pooled specificity and sensitivity of $F \geq 1$.

Table I. Quality assessment details

\begin{tabular}{|c|c|c|c|c|c|c|c|}
\hline \multirow[t]{2}{*}{ Studies } & \multicolumn{4}{|c|}{ Risk of bias } & \multicolumn{3}{|c|}{ Applicability concerns } \\
\hline & $\begin{array}{l}\text { Patient } \\
\text { selection }\end{array}$ & $\begin{array}{l}\text { Index } \\
\text { test }\end{array}$ & $\begin{array}{l}\text { Reference } \\
\text { standard }\end{array}$ & $\begin{array}{l}\text { Flow and } \\
\text { timing }\end{array}$ & $\begin{array}{l}\text { Patient } \\
\text { selection }\end{array}$ & $\begin{array}{l}\text { Index } \\
\text { test }\end{array}$ & $\begin{array}{l}\text { Reference } \\
\text { standard }\end{array}$ \\
\hline Zhang 2015 [15] & Low & Low & Unclear & Unclear & Low & Unclear & Unclear \\
\hline Dong 2015 [16] & Low & Low & Unclear & Unclear & Low & Unclear & Unclear \\
\hline Friedrich-Rust 2013 [17] & Low & Low & Unclear & Low & Low & Low & Low \\
\hline Zhang 2013 [18] & Low & Unclear & Unclear & Unclear & Low & Low & Unclear \\
\hline Liu 2015 [19] & Low & Low & Unclear & Unclear & Low & Unclear & Low \\
\hline Li $2014[20]$ & Low & Low & Unclear & Low & Low & Low & Low \\
\hline Nishikawa 2014 [21] & Low & Low & Low & Low & Low & Low & Low \\
\hline Park 2016 [22] & Low & Unclear & Low & Low & Low & Low & Low \\
\hline Silva Junior 2014 [23] & Low & Low & Low & Low & Low & Low & Low \\
\hline Yamada2014 [24] & Low & Low & Low & Low & Low & Low & Low \\
\hline Chen 2015 [25] & Low & Low & Unclear & Unclear & Unclear & Low & Low \\
\hline Chen 2012 [26] & Low & Low & Unclear & Unclear & Unclear & Low & Low \\
\hline Ye 2012[27] & Low & Unclear & Unclear & Unclear & Unclear & Low & Low \\
\hline Takaki 2014 [28] & Low & Low & Low & Low & Low & Low & Low \\
\hline Sporea 2011 [29] & Low & Low & Low & Low & Low & Low & Low \\
\hline Lupsor 2009 [30] & Low & Low & Low & Low & Low & Low & Low \\
\hline Cabassa 2015 [31] & Unclear & Low & Low & Low & Unclear & Low & Low \\
\hline Tai 2015 [32] & Low & Low & Low & Low & Low & Low & Unclear \\
\hline Sporea 2010 [33] & Low & Low & Low & Low & Low & Unclear & Unclear \\
\hline Friedrich-Rust 2009 [34] & Low & Low & Low & Low & Low & Low & Unclear \\
\hline Goertz 2010 [35] & Low & Low & Low & Low & Low & Low & Low \\
\hline
\end{tabular}

the QUADAS-2 tool (Table I). Articles' characteristics are presented in Table II, and the results of the diagnostic tests and ARFI values are listed in Table III.

\section{Analysis of the Results}

We combined the Se and Sp of different fibrosis stages as can be seen in Table IV. The summary sensitivity was 0.79 (95\% CI: $0.76-0.83)$ and the summary specificity was 0.86 (95\% CI: $0.85-0.88)$. The higher the Se, the better the positive predictive efficacy, and similarly, the higher the $\mathrm{Sp}$, the better the negative predictive efficacy.
Thus the results indicated that ARFI elastography has a moderate efficacy in the detection of liver fibrosis and non-liver fibrosis patients in $\mathrm{CHB}$ and $\mathrm{CHC}$ patients. The pooled $\mathrm{Se}$ and $\mathrm{Sp}$ of $\mathrm{F} \geq 1$ were 0.72 (95\% CI: 0.65-0.78, $\left.I^{2}=62.64\right)$ and $0.86\left(95 \% \mathrm{CI}: 0.77-0.93, I^{2}=0.00\right)$, respectively (fig 2). In addition, we found that the combined $\mathrm{Se}$ and $\mathrm{Sp}$ of $\mathrm{F}=2$ were 0.75 (95\% CI: $\left.0.69-0.80, I^{2}=79.62\right)$ and 0.85 (95\% CI: $0.81-0.89, I^{2}=48.53$ ) (fig 3). Se and Sp of $\mathrm{F} \geq 3$ were 0.84 (95\% CI: $\left.0.80-0.88, I^{2}=61.37\right)$ and 0.90 (95\% CI: $\left.0.86-0.92, I^{2}=65.1\right)$ (fig 4). On the oth- 
Acoustic Radiation Force Impulse (ARFI) Elastography for non-invasive evaluation of hepatic fibrosis

Table II. Details of article characteristics concerning the diagnosis test

\begin{tabular}{|c|c|c|c|c|c|c|c|c|c|c|c|c|}
\hline Author, year, country & $\begin{array}{l}\text { Histologi- } \\
\text { cal score }\end{array}$ & Disease & $\begin{array}{l}\text { N, Age [year } \\
\text { (SE)] }\end{array}$ & $\begin{array}{l}\text { Gender } \\
(\mathbf{M} / \mathbf{F})\end{array}$ & $\overline{\mathbf{R}}$ & Stage & $\begin{array}{l}\begin{array}{l}\text { Cut off } \\
(\mathrm{m} / \mathrm{s})\end{array} \\
\end{array}$ & tp+fn & $f p+t n$ & Se & Sp & AUROC \\
\hline Zhang 2015 China [15] & Scheuer & CHB & $\begin{array}{l}180 \\
36.4(10.96)\end{array}$ & $139 / 41$ & 0.599 & $\begin{array}{l}F \geq 2 \\
F \geq 3 \\
F=4\end{array}$ & $\begin{array}{l}1.46 \\
1.59 \\
1.75\end{array}$ & $\begin{array}{l}129 \\
69 \\
33\end{array}$ & $\begin{array}{l}51 \\
111 \\
147\end{array}$ & $\begin{array}{l}0.59 \\
0.71 \\
0.73\end{array}$ & $\begin{array}{l}0.88 \\
0.86 \\
0.84\end{array}$ & $\begin{array}{l}0.764 \\
0.852 \\
0.825\end{array}$ \\
\hline Dong 2015 China [16] & $\mathrm{CPC}^{*}$ & CHB & $\begin{array}{l}83 \\
41(11.4)\end{array}$ & $71 / 10$ & 0.577 & $\begin{array}{l}F \geq 1 \\
F \geq 2 \\
F \geq 3 \\
F=4\end{array}$ & $\begin{array}{l}1.295 \\
1.295 \\
1.54 \\
1.835\end{array}$ & $\begin{array}{l}74 \\
49 \\
24 \\
8\end{array}$ & $\begin{array}{l}9 \\
34 \\
59 \\
75\end{array}$ & $\begin{array}{l}0.683 \\
0.829 \\
0.762 \\
0.667\end{array}$ & $\begin{array}{l}0.8 \\
0.65 \\
0.9 \\
0.855\end{array}$ & $\begin{array}{l}0.72 \\
0.762 \\
0.884 \\
0.723\end{array}$ \\
\hline $\begin{array}{l}\text { Friedrich- } \\
\text { Rust } 2013 \text { Germany, } \\
\text { Holland [17] }\end{array}$ & Metavir & $\mathrm{CHB}$ & $\begin{array}{l}114 \\
39(12)\end{array}$ & $77 / 37$ & 0.415 & $\begin{array}{l}F \geq 1 \\
F \geq 2 \\
F \geq 3 \\
F=4\end{array}$ & $\begin{array}{l}- \\
- \\
- \\
-\end{array}$ & $\begin{array}{l}81 \\
36 \\
13 \\
5\end{array}$ & $\begin{array}{l}33 \\
83 \\
101 \\
109\end{array}$ & $\begin{array}{l}- \\
- \\
-\end{array}$ & $\begin{array}{l}- \\
- \\
-\end{array}$ & $\begin{array}{l}0.66 \\
0.73 \\
0.94 \\
0.97\end{array}$ \\
\hline Zhang 2013 China [18] & $\mathrm{CPC}^{*}$ & $\mathrm{CHC}$ & $\begin{array}{l}108 \\
44.2(13.3)\end{array}$ & $54 / 54$ & 0.61 & $\begin{array}{l}F \geq 2 \\
F \geq 3 \\
F=4\end{array}$ & $\begin{array}{l}1.529 \\
1.78 \\
1.797\end{array}$ & $\begin{array}{l}72 \\
41 \\
14\end{array}$ & $\begin{array}{l}36 \\
67 \\
94\end{array}$ & $\begin{array}{l}0.569 \\
0.732 \\
0.786\end{array}$ & $\begin{array}{l}0.889 \\
0.925 \\
0.745\end{array}$ & $\begin{array}{l}0.779 \\
0.863 \\
0.79\end{array}$ \\
\hline Liu 2015 [19 China] & Metavir & CHB & $\begin{array}{l}108 \\
39.8(9.7)\end{array}$ & $81 / 27$ & 0.63 & $\begin{array}{l}\mathrm{F} \geq 2 \\
\mathrm{~F}=4\end{array}$ & $\begin{array}{l}1.27 \\
1.65\end{array}$ & $\begin{array}{l}66 \\
29\end{array}$ & $\begin{array}{l}42 \\
79\end{array}$ & $\begin{array}{l}0.84 \\
0.931\end{array}$ & $\begin{array}{l}0.831 \\
0.768\end{array}$ & - \\
\hline Li 2014 China [20] & Metavir & $\mathrm{CHC}$ & $\begin{array}{l}128 \\
69.1(4.7)\end{array}$ & $86 / 42$ & 0.649 & $\begin{array}{l}F \geq 2 \\
F \geq 3 \\
F=4\end{array}$ & $\begin{array}{l}1.53 \\
1.79 \\
1.789\end{array}$ & $\begin{array}{l}87 \\
47 \\
17\end{array}$ & $\begin{array}{l}41 \\
81 \\
111\end{array}$ & $\begin{array}{l}0.576 \\
0.764 \\
0.789\end{array}$ & $\begin{array}{l}0.895 \\
0.965 \\
0.765\end{array}$ & $\begin{array}{l}0.775 \\
0.901 \\
0.792\end{array}$ \\
\hline $\begin{array}{l}\text { Nishikawa } 2014 \text { Japan } \\
{[21]}\end{array}$ & Metavir & $\mathrm{CHC}$ & $\begin{array}{l}108 \\
59.5(12.6)\end{array}$ & $56 / 52$ & 0.872 & $\begin{array}{l}F \geq 1 \\
F \geq 2 \\
F \geq 3 \\
F=4\end{array}$ & $\begin{array}{l}1.28 \\
1.28 \\
1.44 \\
1.73\end{array}$ & $\begin{array}{l}94 \\
77 \\
45 \\
14\end{array}$ & $\begin{array}{l}14 \\
31 \\
63 \\
94\end{array}$ & $\begin{array}{l}0.691 \\
0.818 \\
0.889 \\
0.857\end{array}$ & $\begin{array}{l}0.857 \\
0.871 \\
0.825 \\
0.862\end{array}$ & $\begin{array}{l}0.81 \\
0.909 \\
0.869 \\
0.885\end{array}$ \\
\hline Park 2016 Korea [22] & Ludwig & CHB & $\begin{array}{l}105 \\
47\end{array}$ & $73 / 32$ & - & $\begin{array}{l}F \geq 2 \\
F \geq 3 \\
F=4\end{array}$ & $\begin{array}{l}- \\
- \\
-\end{array}$ & $\begin{array}{l}78 \\
51 \\
30\end{array}$ & $\begin{array}{l}27 \\
54 \\
75\end{array}$ & $\begin{array}{l}- \\
- \\
-\end{array}$ & $\begin{array}{l}- \\
- \\
-\end{array}$ & $\begin{array}{l}0.814 \\
0.848 \\
0.752\end{array}$ \\
\hline $\begin{array}{l}\text { Silva Junior } 2014 \\
\text { Brazil [23] }\end{array}$ & Metavir & $\mathrm{CHC}$ & $\begin{array}{l}51 \\
53.8(1.53)\end{array}$ & $18 / 33$ & 0.833 & $\begin{array}{l}F \geq 1 \\
F \geq 2 \\
F \geq 3 \\
F=4\end{array}$ & $\begin{array}{l}1.19 \\
1.31 \\
1.68 \\
1.95\end{array}$ & $\begin{array}{l}43 \\
28 \\
18 \\
9\end{array}$ & $\begin{array}{l}8 \\
23 \\
33 \\
42\end{array}$ & $\begin{array}{l}0.884 \\
0.893 \\
0.944 \\
1\end{array}$ & $\begin{array}{l}0.75 \\
0.87 \\
0.909 \\
0.952\end{array}$ & $\begin{array}{l}0.88 \\
0.9 \\
0.97 \\
0.98\end{array}$ \\
\hline $\begin{array}{l}\text { Yamada } 2014 \text { Japan } \\
\text { [24] }\end{array}$ & Metavir & $\mathrm{CHC}$ & $\begin{array}{l}124 \\
57.0(12.1)\end{array}$ & $56 / 68$ & 0.764 & $\begin{array}{l}\mathrm{F} \geq 2 \\
\mathrm{~F} \geq 3\end{array}$ & $\begin{array}{l}1.26 \\
1.46\end{array}$ & $\begin{array}{l}40 \\
26\end{array}$ & $\begin{array}{l}84 \\
98\end{array}$ & $\begin{array}{l}0.925 \\
0.846\end{array}$ & $\begin{array}{l}0.762 \\
0.878\end{array}$ & $\begin{array}{l}0.89 \\
0.943\end{array}$ \\
\hline Chen 2015 Taiwan [25] & Metavir & $\mathrm{CHC}$ & $\begin{array}{l}137 \\
54\end{array}$ & $63 / 74$ & 0.593 & $\begin{array}{l}F \geq 2 \\
F \geq 3 \\
F=4\end{array}$ & $\begin{array}{l}1.59 \\
1.73 \\
1.96\end{array}$ & $\begin{array}{l}103 \\
58 \\
24\end{array}$ & $\begin{array}{l}34 \\
79 \\
113\end{array}$ & $\begin{array}{l}0.728 \\
0.914 \\
1\end{array}$ & $\begin{array}{l}0.794 \\
0.772 \\
0.681\end{array}$ & $\begin{array}{l}0.9349 \\
0.8997 \\
0.8647\end{array}$ \\
\hline Chen 2012 Taiwan [26] & Metavir & $\mathrm{CHC}$ & 127 & $59 / 68$ & 0.696 & $\begin{array}{l}F \geq 2 \\
F \geq 3 \\
F=4\end{array}$ & $\begin{array}{l}1.55 \\
1.18 \\
1.98\end{array}$ & $\begin{array}{l}81 \\
41 \\
18\end{array}$ & $\begin{array}{l}46 \\
86 \\
109\end{array}$ & $\begin{array}{l}0.741 \\
0.902 \\
0.889\end{array}$ & $\begin{array}{l}0.87 \\
0.895 \\
0.798\end{array}$ & $\begin{array}{l}0.847 \\
0.902 \\
0.831\end{array}$ \\
\hline Ye 2011 China [27] & Metavir & $\mathrm{CHB}$ & $\begin{array}{l}264 \\
39.3(13.7)\end{array}$ & $158 / 106$ & 0.87 & $\begin{array}{l}F \geq 3 \\
F=4\end{array}$ & $\begin{array}{l}1.69 \\
1.88\end{array}$ & $\begin{array}{l}164 \\
141\end{array}$ & $\begin{array}{l}100 \\
123\end{array}$ & $\begin{array}{l}0.939 \\
0.957\end{array}$ & $\begin{array}{l}0.95 \\
0.918\end{array}$ & $\begin{array}{l}0.99 \\
0.97\end{array}$ \\
\hline Takaki 2014 Japan [28] & Metavir & $\mathrm{CHC}$ & $\begin{array}{l}176 \\
61.2(11.94)\end{array}$ & $84 / 92$ & 0.725 & $\begin{array}{l}F \geq 2 \\
F \geq 3 \\
F=4\end{array}$ & $\begin{array}{l}1.25 \\
1.595 \\
1.775\end{array}$ & $\begin{array}{l}128 \\
37 \\
12\end{array}$ & $\begin{array}{l}37 \\
128 \\
153\end{array}$ & $\begin{array}{l}0.75 \\
0.849 \\
0.856\end{array}$ & $\begin{array}{l}0.781 \\
0.815 \\
0.889\end{array}$ & $\begin{array}{l}0.773 \\
0.863 \\
0.915\end{array}$ \\
\hline $\begin{array}{l}\text { Sporea } 2011 \text { Romania } \\
\text { [29] }\end{array}$ & Metavir & $\mathrm{CHC}$ & $\begin{array}{l}274 \\
50.1(11.8)\end{array}$ & $113 / 161$ & 0.727 & $\begin{array}{l}F \geq 1 \\
F \geq 2 \\
F \geq 3 \\
F=4\end{array}$ & $\begin{array}{l}1.19 \\
1.21 \\
1.58 \\
1.82\end{array}$ & $\begin{array}{l}233 \\
190 \\
116 \\
65\end{array}$ & $\begin{array}{l}29 \\
72 \\
146 \\
197\end{array}$ & $\begin{array}{l}0.73 \\
0.84 \\
0.84 \\
0.91\end{array}$ & $\begin{array}{l}0.93 \\
0.91 \\
0.94 \\
0.9\end{array}$ & $\begin{array}{l}0.88 \\
0.893 \\
0.908 \\
0.937\end{array}$ \\
\hline $\begin{array}{l}\text { Lupsor } 2009 \text { Romania } \\
\text { [30] }\end{array}$ & Metavir & $\mathrm{CHC}$ & $\begin{array}{l}112 \\
48.9(12.28)\end{array}$ & $45 / 67$ & 0.717 & $\begin{array}{l}F \geq 1 \\
F \geq 2 \\
F \geq 3 \\
F=4\end{array}$ & $\begin{array}{l}1.19 \\
1.34 \\
1.61 \\
2\end{array}$ & $\begin{array}{l}92 \\
63 \\
45 \\
38\end{array}$ & $\begin{array}{l}14 \\
43 \\
61 \\
68\end{array}$ & $\begin{array}{l}0.621 \\
0.678 \\
0.791 \\
0.8\end{array}$ & $\begin{array}{l}0.857 \\
0.929 \\
0.948 \\
0.955\end{array}$ & $\begin{array}{l}0.725 \\
0.869 \\
0.9 \\
0.936\end{array}$ \\
\hline Cabassa 2015 Italy [31] & Knodell & $\mathrm{B} \& \mathrm{C}$ & 84 & $40 / 44$ & - & $\mathrm{F} \geq 3$ & 2.11 & 26 & 58 & 0.73 & 0.92 & 0.879 \\
\hline Tai 2015 Taiwan [32] & Metavir & $\begin{array}{l}\text { CHB } \\
\text { CHC }\end{array}$ & $\begin{array}{l}121,48(11) \\
83,53(11)\end{array}$ & $\begin{array}{l}98 / 23 \\
48 / 35\end{array}$ & - & $\begin{array}{l}F=4 \\
F=4\end{array}$ & $\begin{array}{l}1.35 \\
1.41\end{array}$ & $\begin{array}{l}43 \\
17\end{array}$ & $\begin{array}{l}74 \\
66\end{array}$ & $\begin{array}{l}0.628 \\
0.706\end{array}$ & $\begin{array}{l}0.705 \\
0.803\end{array}$ & $\begin{array}{l}0.681 \\
0.802\end{array}$ \\
\hline $\begin{array}{l}\text { Sporea } 2010 \text { Romania } \\
\text { [33] }\end{array}$ & Metavir & $\mathrm{B} \& \mathrm{C}$ & $\begin{array}{l}71 \\
50.7(12.9)\end{array}$ & $41 / 30$ & - & $\begin{array}{l}\mathrm{F} \geq 2 \\
\mathrm{~F}=4\end{array}$ & $\begin{array}{l}1.33 \\
1.8\end{array}$ & $\begin{array}{l}65 \\
16\end{array}$ & $\begin{array}{l}6 \\
55\end{array}$ & $\begin{array}{l}0.71 \\
1\end{array}$ & $\begin{array}{l}0.66 \\
0.77\end{array}$ & $\begin{array}{l}0.649 \\
0.868\end{array}$ \\
\hline $\begin{array}{l}\text { Friedrich-Rust } 2009 \\
\text { Germany [34] }\end{array}$ & Metavir & $\mathrm{B} \& \mathrm{C}$ & $\begin{array}{l}81 \\
48(14)\end{array}$ & $46 / 35$ & 0.71 & $\begin{array}{l}F \geq 2 \\
F \geq 3 \\
F=4\end{array}$ & $\begin{array}{l}1.37 \\
1.45 \\
1.75\end{array}$ & $\begin{array}{l}54 \\
31 \\
22\end{array}$ & $\begin{array}{l}27 \\
50 \\
59\end{array}$ & $\begin{array}{l}68.5 \\
83.9 \\
81.8\end{array}$ & $\begin{array}{l}92.6 \\
86 \\
91.5\end{array}$ & $\begin{array}{l}- \\
-\end{array}$ \\
\hline $\begin{array}{l}\text { Goertz } 2012 \text { Germany } \\
\text { [35] }\end{array}$ & Ludwig & $\mathrm{B} \& \mathrm{C}$ & $\begin{array}{l}57 \\
45(11)\end{array}$ & $30 / 27$ & 0.64 & $\begin{array}{l}F \geq 2 \\
F \geq 3 \\
F=4\end{array}$ & $\begin{array}{l}- \\
- \\
-\end{array}$ & $\begin{array}{l}30 \\
16 \\
12\end{array}$ & $\begin{array}{l}27 \\
41 \\
45\end{array}$ & - & - & $\begin{array}{l}0.85 \\
0.92 \\
0.87\end{array}$ \\
\hline
\end{tabular}

*Chinese Program of Prevention and Cure for Viral Hepatitis, N: number of patients, SE: standard error, M/F: Male/Female, B\&C: CHB and CHC. F: acronym of the fibrosis stages. 
Table III. Details of article characteristics for ARFI values

\begin{tabular}{|c|c|c|c|c|}
\hline Author & Disease & Stage & $\mathbf{n}$ & ARFI \\
\hline \multirow[t]{4}{*}{ Zhang [15] } & $\mathrm{CHB}$ & F0-1 & 51 & 1.24 \\
\hline & & F2 & 60 & 1.4 \\
\hline & & F3 & 36 & 1.93 \\
\hline & & F4 & 33 & 2.19 \\
\hline \multirow[t]{4}{*}{ Zhang [18] } & $\mathrm{CHC}$ & F0-1 & 36 & 1.26 \\
\hline & & $\mathrm{F} 2$ & 31 & 1.45 \\
\hline & & F3 & 27 & 2.01 \\
\hline & & $\mathrm{F} 4$ & 14 & 2.28 \\
\hline \multirow[t]{4}{*}{ Li [20] } & $\mathrm{CHC}$ & F0-1 & 41 & 1.23 \\
\hline & & $\mathrm{F} 2$ & 40 & 1.48 \\
\hline & & F3 & 30 & 2.06 \\
\hline & & $\mathrm{F} 4$ & 17 & 2.3 \\
\hline \multirow[t]{4}{*}{ Park [22] } & $\mathrm{CHB}$ & F0-1 & 27 & 1.38 \\
\hline & & F2 & 27 & 1.61 \\
\hline & & F3 & 21 & 2.17 \\
\hline & & F4 & 30 & 2.4 \\
\hline \multirow[t]{4}{*}{ Chen [26] } & $\mathrm{CHC}$ & F0-1 & 46 & 1.283 \\
\hline & & $\mathrm{F} 2$ & 40 & 1.63 \\
\hline & & F3 & 23 & 2.433 \\
\hline & & $\mathrm{F} 4$ & 18 & 2.619 \\
\hline \multirow[t]{5}{*}{ Ye [27] } & CHB & F0-1 & 60 & 1.13 \\
\hline & & F0-1 & 17 & 1.27 \\
\hline & & F2 & 23 & 1.5 \\
\hline & & F3 & 23 & 1.85 \\
\hline & & F4 & 141 & 2.5 \\
\hline \multirow[t]{4}{*}{ Takaki [28] } & $\mathrm{CHC}$ & $\mathrm{F} 1$ & 37 & 1.165 \\
\hline & & F2 & 91 & 1.315 \\
\hline & & F3 & 25 & 1.75 \\
\hline & & $\mathrm{F} 4$ & 12 & 2.55 \\
\hline \multirow[t]{5}{*}{ Sporea [29] } & $\mathrm{CHC}$ & F0-1 & 29 & 0.97 \\
\hline & & F0-1 & 43 & 1.07 \\
\hline & & F2 & 74 & 1.33 \\
\hline & & F3 & 51 & 1.71 \\
\hline & & F4 & 65 & 2.6 \\
\hline \multirow[t]{5}{*}{ Lupsor [30] } & $\mathrm{CHC}$ & F0-1 & 14 & 1.103 \\
\hline & & F0-1 & 29 & 1.067 \\
\hline & & $\mathrm{F} 2$ & 18 & 1.504 \\
\hline & & F3 & 7 & 1.52 \\
\hline & & F4 & 38 & 2.552 \\
\hline \multirow[t]{9}{*}{ Sporea [33] } & CHB & F0-1 & 8 & 1.24 \\
\hline & & F2 & 25 & 1.39 \\
\hline & & F3 & 12 & 1.6 \\
\hline & & F4 & 5 & 2.39 \\
\hline & $\mathrm{CHC}$ & F0-1 & 6 & 1.14 \\
\hline & & F0-1 & 9 & 1.16 \\
\hline & & $\mathrm{F} 2$ & 37 & 1.33 \\
\hline & & F3 & 31 & 1.55 \\
\hline & & $\mathrm{F} 4$ & 13 & 2.46 \\
\hline \multirow[t]{8}{*}{ Goertz [35] } & CHB & F0-1 & 14 & 1.24 \\
\hline & & F2 & 2 & 1.42 \\
\hline & & F3 & 2 & 1.87 \\
\hline & & F4 & 3 & 2.67 \\
\hline & $\mathrm{CHC}$ & F0-1 & 13 & 1.53 \\
\hline & & F2 & 12 & 1.5 \\
\hline & & F3 & 2 & 3.79 \\
\hline & & F4 & 9 & 2.38 \\
\hline
\end{tabular}

$\mathrm{N}$ : number of patients, . F: acronym of the fibrosis stages.

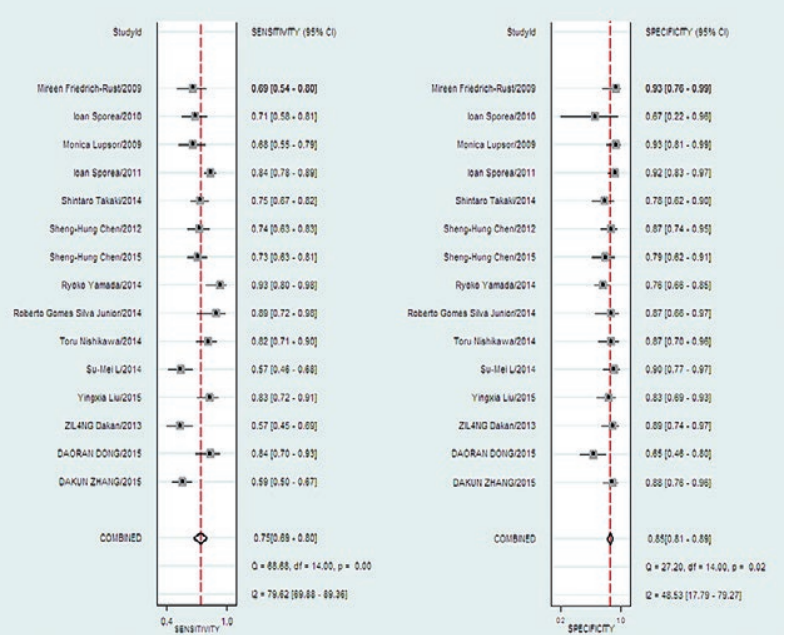

Fig 3. Pooled specificity and sensitivity of $F \geq 2$.

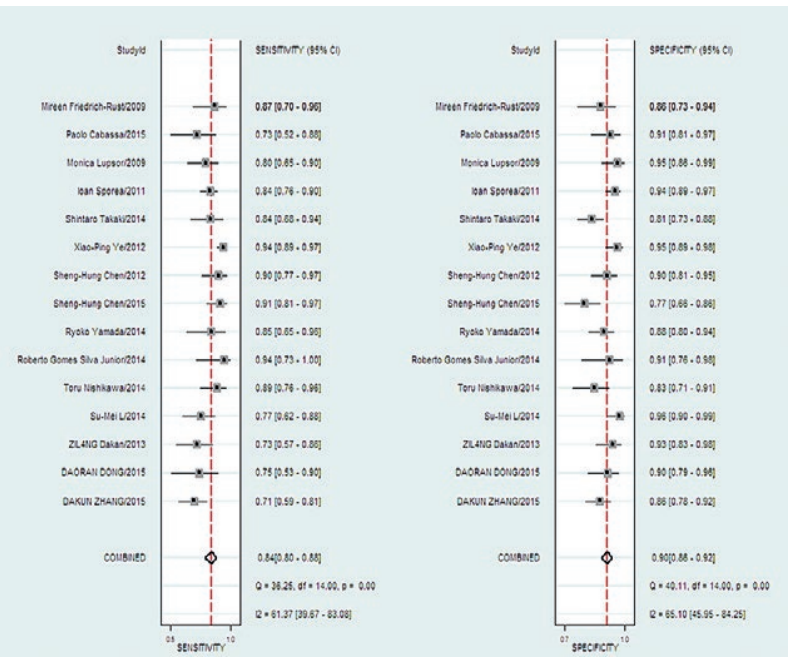

Fig 4. Pooled specificity and sensitivity of $F \geq 3$.

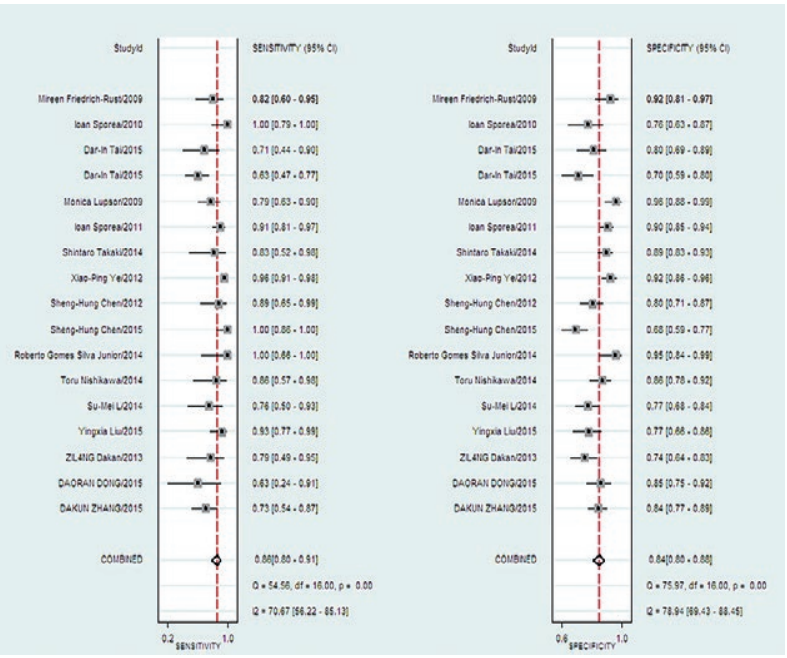

Fig 5. Pooled specificity and sensitivity of $F=4$. 
er hand, the pooled $\mathrm{Se}$ and $\mathrm{Sp}$ of $\mathrm{F}=4$ were $0.86(95 \%$ CI: $\left.0.80-0.91, I^{2}=70.67\right)$ and 0.84 (95\% CI: $0.80-0.88$, $I^{2=} 78.94$ ), respectively (fig 5). The separate analysis results of different liver fibrosis stages also showed a good predictive value of ARFI elastography in liver fibrosis examinations especially in stage $\mathrm{F} \geq 3$ and $\mathrm{F}=4$ since the $\mathrm{Se}$ and $\mathrm{Sp}$ were relatively higher than other stages. To determine the source of heterogeneity, we used metaregression and included in the model different parameters, including country of origin, age group, histological evaluation approach, patients' number, disease type, and the respective cut-off values. However, no statistical significance was established except for the cut-off value of $\mathrm{F} \geq 2$ in sensitivity (coef $=0.6, \mathrm{p}=0.004$ ) (fig 6).

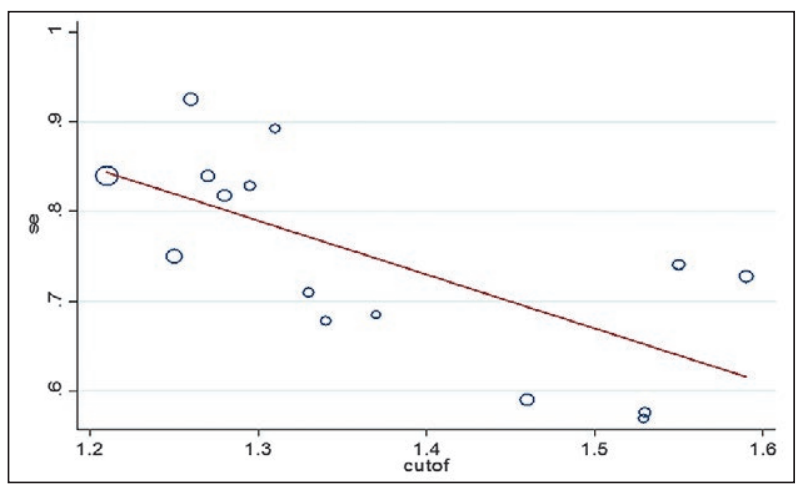

Fig 6. Meta-regression of $\mathrm{F} \geq 2$ for sensitivity.

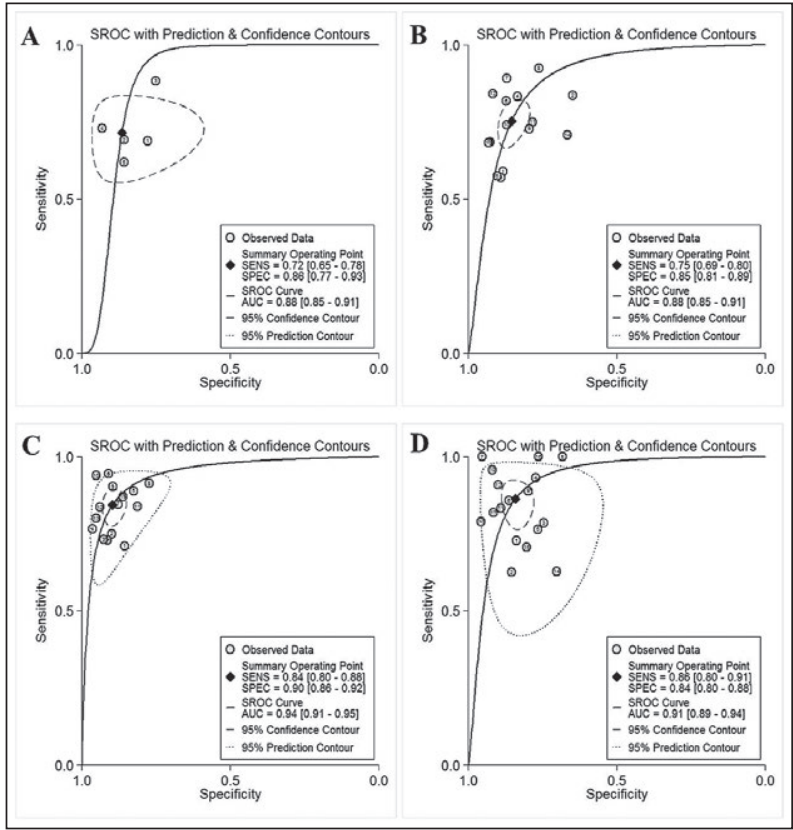

Fig 7. ROC curves of different fibrosis stage. A: ROC curve of $\mathrm{F} \geq 1$; $\mathrm{B}$ : ROC curve of $\mathrm{F} \geq 2$ : $\mathrm{C}$ : ROC curve of $\mathrm{F} \geq 3$; $\mathrm{D}$ : ROC curve of $\mathrm{F}=4$.
We also obtained the ROC curves of the different fibrosis stages as the comprehensive indicator to reflect diagnosis efficacy of ARFI elastography. The larger the area under the ROC curves, the greater the diagnosis accuracy. As is shown in figure 7, the following values were obtained: for $\mathrm{F} \geq 1$, AUROC $=0.88,95 \% \mathrm{CI}$ : $0.85-0.91$; for $\mathrm{F} \geq 2$, AUROC $=0.88,95 \%$ CI: $0.85-0.91$ $\mathrm{r}$ for $\mathrm{F} \geq 3$, AUROC $=0.94,95 \% \mathrm{CI}$ : $0.91-0.95$; and for $\mathrm{F}=4$, AUROC $=0.91,95 \%$ CI: $0.89-0.94$. The results indicated that the area under ROC curves were high especially in stages $\mathrm{F} \geq 3$ and $\mathrm{F}=4$, which indicate that ARFI elastography has a good diagnosis accuracy in liver fibrosis examinations especially in stage $\mathrm{F} \geq 3$ and $\mathrm{F}=4$.

Table IV. Pooled diagnostic test characteristics

\begin{tabular}{lllll}
\hline Effect & Stage & $\begin{array}{l}\text { Estimate } \\
\text { result (\%) }\end{array}$ & $\mathbf{9 5 \%}$ CI & I2 \\
\hline Sensitivity & $\mathrm{F} \geq 1$ & 0.72 & $0.65-0.78$ & 62.64 \\
$(0.79 ; 95 \%$ CI: & $\mathrm{F} \geq 2$ & 0.75 & $0.69-0.80$ & 79.62 \\
$0.76-0.83)$ & $\mathrm{F} \geq 3$ & 0.84 & $0.80-0.88$ & 61.37 \\
& $\mathrm{~F}=4$ & 0.86 & $0.80-0.91$ & 70.67 \\
& & & & \\
Specificity & $\mathrm{F} \geq 1$ & 0.86 & $0.77-0.93$ & 0.0 \\
$(0.86 ; 95 \%$ CI: & $\mathrm{F} \geq 2$ & 0.85 & $0.81-0.89$ & 48.53 \\
$0.85-0.88)$ & $\mathrm{F} \geq 3$ & 0.90 & $0.86-0.92$ & 65.10 \\
& $\mathrm{~F}=4$ & 0.84 & $0.80-0.88$ & 78.94 \\
\hline
\end{tabular}

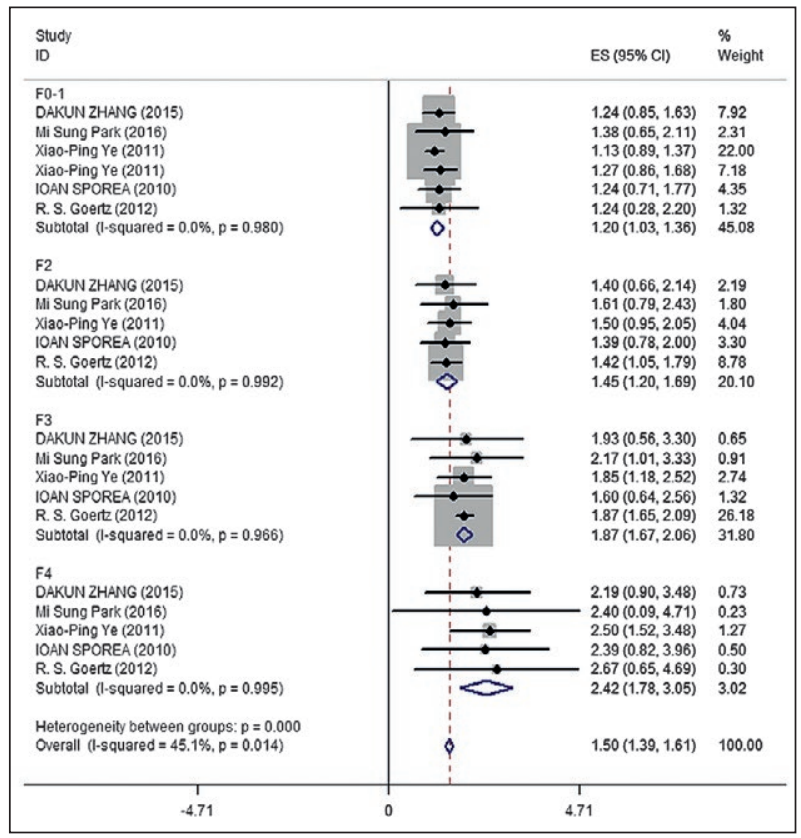

Fig 8. Forest plot for ARFI values of CHB in different stages. 
We combined the ARFI values of $\mathrm{CHB}$ and $\mathrm{CHC}$ patients in different stages and found that $\mathrm{CHC}$ patients had higher ARFI values, especially in the F3 stage, 1.87 (95\% CI: 1.67, 2.06, fig 8) for CHB and 2.31 (95\% CI: 2.09 , 2.52, fig 9) for CHC. There was no report bias as indicated by the funnel plot results which are illustrated in figure 10 .

\section{Discussions}

LB is the "golden" standard to evaluate the stages of liver fibrosis, but its application poses a risk of complications and bias. Thus, ARFI elastography has been researched and used widely, as a promising alternative non-

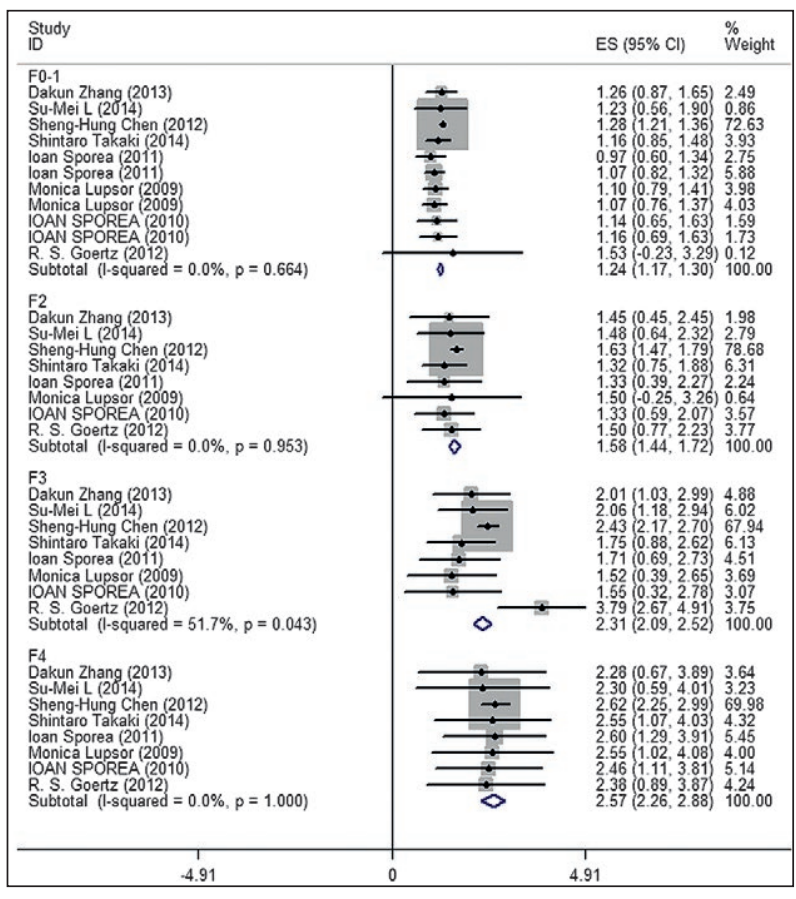

Fig 9. Forest plot for ARFI values of CHB in different stages.

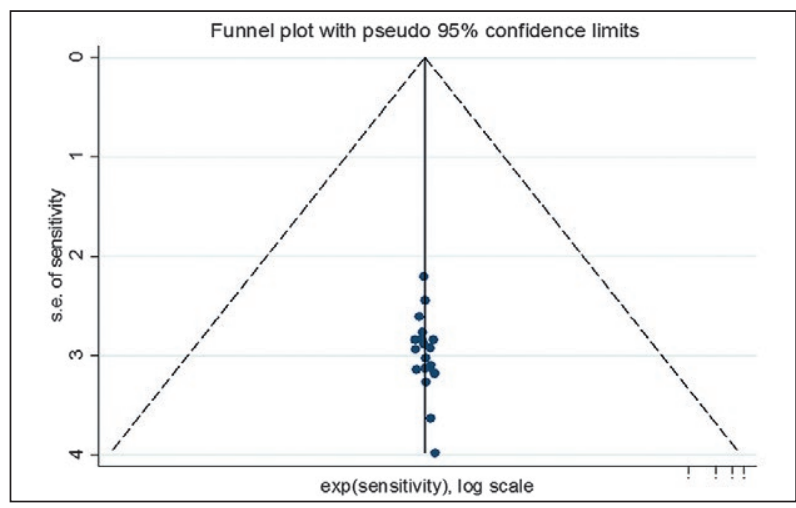

Fig 10. Funnel plot of the diagnosis test. invasive method [36]. In the current systematic review and meta-analysis, we evaluated the accuracy of ARFI elastography in patients with $\mathrm{CHB}$ and $\mathrm{CHC}$. The composite sensitivity $=0.79(95 \%$ CI: $0.76-0.83)$ and specificity $=0.86(95 \%$ CI: $0.85-0.88)$ were presented which indicates that ARFI elastography has a moderately good efficacy in positive and negative predictive values. The separated analysis results of stages indicate that ARFI elastography has a moderately good efficacy in positive and negative predictive values in different stages (reflected from Se and $\mathrm{Sp}$ ) and has a moderate accuracy in diagnosis of stage $\mathrm{F} \geq 1$ and $\mathrm{F} \geq 2(\mathrm{~F} \geq 1$ : $\mathrm{Se}=0.72,95 \% \mathrm{CI}$ : $0.65-0.78 ; \mathrm{Sp}=0.86,95 \%$ CI: $0.77-0.93$; AUROC $=0.88$, 95\% CI: $0.85-0.91$; and $\mathrm{F} \geq 2$ : $\mathrm{Se}=0.75$. 95\% CI: 0.69 0.80 ; $\mathrm{Sp}=0.85,95 \%$ CI: $0.81-0.89$; AUROC $=0.88,95 \%$ CI: $0.85-0.91)$ and good diagnosis accuracy for stage $\mathrm{F} \geq 3$ and $\mathrm{F}=4$ reflected through AUROC values (AUROC $=0.94,95 \%$ CI: $0.91-0.95$ and $A U R O C=0.91,95 \%$ CI: $0.89-0.94$ of $\mathrm{F} \geq 3$ and $\mathrm{F}=4$, respectively).

Our study results demonstrate that ARFI elastography is moderately accurate which is consistent with the findings of Bota et al [37] indicating that ARFI is a suitable method for assessing liver fibrosis. Moreover, our results also showed that ARFI elastography has a better ability to evaluate higher-stage liver fibrosis and liver cirrhosis ( $\mathrm{F}=3$ and $\mathrm{F}=4$, respectively). When diagnosing a higher stage of these diseases, especially for liver fibrosis over stage 3 ( $F \geq 3)$, ARFI elastography has a higher sensitivity, specificity, and AUROC values than $F \geq 2$ and $F \geq 1$ liver fibrosis stage. In addition, ARFI elastography also shows a better efficacy in the diagnosis of liver cirrhosis ( $F=4$ ), than in that of stages $F \geq 2$ and $F \geq 1$ of liver fibrosis, which is not consistent with the conclusions of Nierhoff et al [12]. Their meta-analysis revealed that ARFI had an excellent diagnostic accuracy for $\mathrm{F}=4$ [12], whereas we found that ARFI showed excellent diagnostic accuracy for $\mathrm{F} \geq 3$ but not for $\mathrm{F}=4$. This discrepancy may have derived from our selection of the population of patients with liver fibrosis that were induced by $\mathrm{CHC}$ and $\mathrm{CHB}$, but the population studied by Nierhoff et al included patients with all types of liver fibrosis.

Intriguingly, the combined ARFI values indicated that $\mathrm{CHC}$ patients had higher ARFI values in all stages, especially in the F3 stage (ARFI value $=1.87,95 \% \mathrm{CI}$ : 1.67-2.06 and ARFI value $=2.31,95 \%$ CI: $2.09-2.52$ for $\mathrm{CHB}$ and $\mathrm{CHC}$, respectively). Perhaps future studies will elucidate the cause of such a difference.

In recent years, many studies have focused on scan probes to evaluate the accuracy of ARFI elastography in liver fibrosis. Cassinotto et al [38] conducted a study aimed at comparing the efficacy of FibroScan M, XLProbes, and ARFI elastography in patients with chronic 
liver disease. He found that ARFI elastography had a lower failure rate and was exceedingly reliable. Potthoff et al [39] discovered that ARFI elastography linear and curve probes exhibited a similar accuracy in the diagnosis of liver fibrosis, but the linear probe had higher ARFI values. It is worth mentioning that to investigate the influence factors, such as interquartile range, success rate, and ARFI performed location, on ARFI elastography efficacy, Bota et al [40] performed an analysis and found that the best correlation between ARFI and fibrosis was obtained at an interquartile range of $<30 \%$ with a success rate of $\geq 60 \%$.

ARFI elastography in the diagnosis of liver fibrosis in HIV-HCV co-infection patients is also an important issue. In an earlier investigation, Frulio et al [41] evaluated the application of ARFI elastography in HIV-HCV co-infection patients and established that ARFI showed promising results in the assessment of morphology and fibrosis during the same session. Natsuda et al [42] also verified that ARFI elastography was useful in staging liver fibrosis in patients with HIV-HCV co-infection.

Our meta-analysis has three strengths. Firstly, to the best of our knowledge, our work is the first meta-analysis to evaluate the accuracy of ARFI elastography in patients with $\mathrm{CHB}$ and $\mathrm{CHC}$; there was no published meta-analysis on this subject until the time of manuscript preparation. Secondly, we found that ARFI elastography is better in the assessment of higher liver fibrosis stages and liver cirrhosis than in the lower liver fibrosis stages. Thirdly, in our analysis, we pooled the latest published studies including a total of 2,691 patients.

Nevertheless, our work has also some limitations. We were not able to completely solve the problem with heterogeneity. In addition, although we exerted maximum efforts to obtain all articles available, we still could not obtain grey articles or articles which were not in English or Chinese.

\section{Conclusion}

ARFI elastography is an accurate and reliable method for examination in patients with liver fibrosis induced by CHB and CHC. Importantly, this approach is especially valuable in the evaluation of higher-stage liver fibrosis and liver cirrhosis. CHC patients have higher ARFI values than CHB patients, especially in the F3 stage.

\section{Conflict of interest: none}

Acknowledgements: This study was supported by the National Natural Science Foundation of China (grant no. 81401425) and the Project of High-level
Personnel in the Beijing Health System (grant no. Z201503101210120051).

\section{References}

1. Liaw YF, Chu CM. Hepatitis B virus infection. Lancet 2009;373:582-592.

2. Bedre RH, Raj U, Misra SP, Varadwaj PK. Antiviral therapy with nucleotide/nucleoside analogues in chronic hepatitis B: A meta-analysis of prospective randomized trials. Indian J Gastroenterol 2016;35:75-82.

3. Busch K, Thimme R. Natural history of chronic hepatitis B virus infection. Med Microbiol Immunol 2015;204:5-10.

4. Jadoul M, Horsmans Y. Towards eradication of hepatitis $\mathrm{C}$ virus from dialysis units. Lancet 2015;386:1514-1515.

5. Manns M, Marcellin P, Poordad F, et al. Simeprevir with pegylated interferon alfa $2 \mathrm{a}$ or $2 \mathrm{~b}$ plus ribavirin in treatment-naive patients with chronic hepatitis $\mathrm{C}$ virus genotype 1 infection (QUEST-2): a randomised, double-blind, placebo-controlled phase 3 trial. Lancet 2014;384:414-426.

6. Lok AS. Chronic hepatitis B. N Engl J Med 2002;346:16821683.

7. Palumbo E. Treatment for chronic hepatitis $\mathrm{C}$ in children: a review. Am J Ther 2009;16:446-450.

8. Rukunuzzaman M, Karim MB. Chronic Hepatitis B in Children - A Review. Mymensingh Med J 2015;24:649-656.

9. Sebastiani G, Gkouvatsos K, Pantopoulos K. Chronic hepatitis $\mathrm{C}$ and liver fibrosis. World J Gastroenterol 2014;20:11033-11053

10. Bota S, Sporea I, Sirli R, Popescu A, Jurchis A. Factors which influence the accuracy of acoustic radiation force impulse (ARFI) elastography for the diagnosis of liver fibrosis in patients with chronic hepatitis C. Ultrasound Med Biol 2013;39:407-412.

11. Nalbantoglu IL, Brunt EM. Role of liver biopsy in nonalcoholic fatty liver disease. World $\mathrm{J}$ Gastroenterol 2014;20:9026-9037.

12. Nierhoff J, Chavez Ortiz AA, Herrmann E, Zeuzem S, Friedrich-Rust M. The efficiency of acoustic radiation force impulse imaging for the staging of liver fibrosis: a metaanalysis. Eur Radiol 2013;23:3040-3053.

13. D’Onofrio M, Crosara S, De Robertis R, et al. Acoustic radiation force impulse of the liver. World J Gastroenterol 2013;19:4841-4849.

14. Whiting P, Rutjes AW, Reitsma JB, Bossuyt PM, Kleijnen $\mathrm{J}$. The development of QUADAS: a tool for the quality assessment of studies of diagnostic accuracy included in systematic reviews. BMC Med Res Methodol 2003;3:25.

15. Zhang D, Chen M, Wang R, et al. Comparison of acoustic radiation force impulse imaging and transient elastography for non-invasive assessment of liver fibrosis in patients with chronic hepatitis B. Ultrasound Med Biol 2015;41:7-14.

16. Dong DR, Hao MN, Li C, et al. Acoustic radiation force impulse elastography, FibroScan(R), Forns' index and their combination in the assessment of liver fibrosis in patients with chronic hepatitis B, and the impact of inflammatory 
activity and steatosis on these diagnostic methods. Mol Med Rep 2015;11:4174-4182.

17. Friedrich-Rust M, Buggisch P, de Knegt RJ, et al. Acoustic radiation force impulse imaging for non-invasive assessment of liver fibrosis in chronic hepatitis B. J Viral Hepat 2013;20:240-247.

18. Zhang DK, Chen M, Liu Y, et al. Clinical value of aeoustie radiation force impulse imaging for quantitative evaluation of degree of liver fibrosis in chronic hepatltis $\mathrm{C}$ patients. Zhonghua Gan Zang Bing Za Zhi 2013;21:599-603.

19. Liu Y, Dong CF, Yang G, et al. Optimal linear combination of ARFI, transient elastography and APRI for the assessment of fibrosis in chronic hepatitis B. Liver Int 2015;35:816-825.

20. Li SM, Li GX, Fu DM, Wang Y, Dang LQ. Liver fibrosis evaluation by ARFI and APRI in chronic hepatitis C. World J Gastroenterol 2014;20:9528-9533.

21. Nishikawa T, Hashimoto S, Kawabe N, et al. Factors correlating with acoustic radiation force impulse elastography in chronic hepatitis C. World J Gastroenterol 2014;20:12891297.

22. Park MS, Kim SW, Yoon KT, et al. Factors Influencing the Diagnostic Accuracy of Acoustic Radiation Force Impulse Elastography in Patients with Chronic Hepatitis B. Gut Liver 2016;10:275-282.

23. Silva Junior RG, Schmillevitch J, Nascimento Mde F, et al. Acoustic radiation force impulse elastography and serum fibrosis markers in chronic hepatitis C. Scand J Gastroenterol 2014;49:986-992.

24. Yamada R, Hiramatsu N, Oze T, et al. Significance of liver stiffness measurement by acoustic radiation force impulse (ARFI) among hepatitis C patients. J Med Virol 2014;86:241-247.

25. Chen SH, Peng CY, Lai HC, et al. Head-to-Head Comparison between Collagen Proportionate Area and Acoustic Radiation Force Impulse Elastography in Liver Fibrosis Quantification in Chronic Hepatitis C. PLoS One 2015; 10:e0140554.

26. Chen SH, Li YF, Lai HC et al. Effects of patient factors on noninvasive liver stiffness measurement using acoustic radiation force impulse elastography in patients with chronic hepatitis C. BMC Gastroenterol 2012;12:105.

27. Ye XP, Ran HT, Cheng J, et al. Liver and spleen stiffness measured by acoustic radiation force impulse elastography for noninvasive assessment of liver fibrosis and esophageal varices in patients with chronic hepatitis B. J Ultrasound Med 2012;31:1245-1253.

28. Takaki S, Kawakami Y, Miyaki D, et al. Non-invasive liver fibrosis score calculated by combination of virtual touch tissue quantification and serum liver functional tests in chronic hepatitis C patients. Hepatol Res 2014;44:280-287.

29. Sporea I, Sirli R, Bota S, et al. Is ARFI elastography reliable for predicting fibrosis severity in chronic HCV hepatitis? World J Radiol 2011;3:188-193.
30. Lupsor M, Badea R, Stefanescu H, et al. Performance of a new elastographic method (ARFI technology) compared to unidimensional transient elastography in the noninvasive assessment of chronic hepatitis C. Preliminary Results. J Gastrointestin Liver Dis 2009;18:303-310.

31. Cabassa P, Ravanelli M, Rossini A, Contessi G, Almajdalawi R, Maroldi R. Acoustic radiation force impulse quantification of spleen elasticity for assessing liver fibrosis. Abdom Imaging 2015;40:738-744.

32. Tai DI, Tsay PK, Jeng WJ, et al. Differences in liver fibrosis between patients with chronic hepatitis $\mathrm{B}$ and $\mathrm{C}$ : evaluation by acoustic radiation force impulse measurements at 2 locations. J Ultrasound Med 2015;34:813-821.

33. Sporea I, Şirli R, Popescu A, Danilă M. Acoustic Radiation Force Impulse (ARFI) - a new modality for the evaluation of liver fibrosis. Med Ultrason 2010;12:26-31.

34. Friedrich-Rust M, Wunder K, Kriener S, et al. Liver fibrosis in viral hepatitis: noninvasive assessment with acoustic radiation force impulse imaging versus transient elastography. Radiology 2009;252:595-604.

35. Goertz RS, Zopf Y, Jugl V, et al. Measurement of liver elasticity with acoustic radiation force impulse (ARFI) technology: an alternative noninvasive method for staging liver fibrosis in viral hepatitis. Ultraschall Med 2010;31:151-155.

36. Piscaglia F, Salvatore V, Di Donato R, et al. Accuracy of VirtualTouch Acoustic Radiation Force Impulse (ARFI) Imaging for the Diagnosis of Cirrhosis during Liver Ultrasonography. Ultraschall Med 2011;32:167-175.

37. Bota S, Herkner H, Sporea I, et al. Meta-analysis: ARFI elastography versus transient elastography for the evaluation of liver fibrosis. Liver Int 2013;33:1138-1147.

38. 38. Cassinotto C, Lapuyade B, Aït-Ali A, et al. Liver fibrosis: noninvasive assessment with acoustic radiation force impulse elastography-comparison with FibroScan $\mathrm{M}$ and XL probes and FibroTest in patients with chronic liver disease. Radiology 2013;269:283-292.

39. Potthoff A, Attia D, Pischke S, et al. Influence of different frequencies and insertion depths on the diagnostic accuracy of liver elastography by acoustic radiation force impulse imaging (ARFI). Eur J Radiol 2013;82:1207-1212.

40. Bota S, Sporea I, Şirli R, Popescu A, Dănilă M, Şendroiu M. Factors that influence the correlation of Acoustic Radiation Force Impulse (ARFI) elastography with liver fibrosis. Med Ultrason 2011;13:135-140.

41. Frulio N, Trillaud H, Perez P, et al. Acoustic Radiation Force Impulse (ARFI) and Transient Elastography (TE) for evaluation of liver fibrosis in HIV-HCV co-infected patients. BMC Infect Dis 2014;14:405.

42. Natsuda K, Soyama A, Takatsuki M, et al. Acoustic radiation force impulse elastography for liver disease staging in human immunodeficiency virus and hepatitis $\mathrm{C}$ virus coinfection. Nihon Shokakibyo Gakkai Zasshi 2014;111:737742. 